\title{
Isolation of mesenchymal stem-like cells in meningioma specimens
}

\author{
HYO-YEOL LIM ${ }^{1 *}$, KYUNG MIN KIM ${ }^{2 *}$, BO KYUNG KIM ${ }^{1}$, JIN-KYOUNG SHIM $^{1}$, JI-HYUN LEE ${ }^{1}$ \\ YONG-MIN HUH ${ }^{3}$, SE-HOON KIM ${ }^{4}$, EUI-HYUN KIM ${ }^{1}$, EUN-KYUNG PARK ${ }^{1}$, KYU-WON SHIM ${ }^{1}$, \\ JONG HEE CHANG ${ }^{1}$, DONG-SEOK KIM ${ }^{1}$, SUN HO KIM ${ }^{1}$, YONG-KIL HONG ${ }^{5}$, \\ SU-JAE LEE ${ }^{6 * *}$ and SEOK-GU KANG ${ }^{2 * *}$ \\ Departments of ${ }^{1}$ Neurosurgery, ${ }^{2}$ Medical Science, ${ }^{3}$ Radiology and ${ }^{4}$ Pathology, Severance Hospital, \\ Yonsei University College of Medicine; ${ }^{5}$ Department of Neurosurgery, Seoul St. Mary's Hospital, \\ The Catholic University of Korea College of Medicine; ${ }^{6}$ Department of Chemistry, \\ Research Institute for Natural Sciences, Hanyang University, Seoul, Republic of Korea
}

Received June 17, 2013; Accepted July 24, 2013

DOI: $10.3892 /$ ijo.2013.2053

\begin{abstract}
Cells resembling bone marrow mesenchymal stem cells (BM-MSCs) have been isolated from glioma specimens; however, little is known about the existence of mesenchymal stem-like cells (MSLCs) in meningioma. Here, we hypothesized that cells similar to BM-MSCs exist in meningioma specimens and sought to investigate whether these putative meningioma stroma MSLCs (MS-MSLCs) could be isolated. To this end, we cultured fresh meningioma specimens using the same protocols as used previously to isolate BM-MSC. Cultured cells were analyzed for surface markers associated with BM-MSCs by fluorescence-activated cell sorting (FACS) and candidate cells were exposed to mesenchymal differentiation conditions. Possible locations of MS-MSLCs were determined by immunohistochemical analysis of sections of meningioma specimens. Spindle-shaped and, adherent cells similar to BM-MSCs were isolated in 2 of 20 meningioma specimens. FACS analysis showed that the surface markers of MS-MSLCs were similar to those of BM-MSCs and the chosen cells demonstrated an ability to differentiate into osteogenic, adipogenic and chondrogenic cells. The tumorigenicity of MS-MSLCs was tested by injection of these cells into the brain of athymic nude mice; no tumors were subsequently
\end{abstract}

Correspondence to: Professor Seok-Gu Kang, Department of Neurosurgery, Severance Hospital, Yonsei University College of Medicine, 50 Yonsei-ro, Seodaemun-gu, Seoul 120-752, Republic of Korea

E-mail: seokgu9@kornet.net

Dr Su-Jae Lee, Department of Chemistry, Research Institute for Natural Sciences, Hanyang University, 17 Haendang-dong, Seongdong-gu, Seoul 133-791, Republic of Korea

E-mail: sj0420@hanyang.ac.kr

${ }^{*}$ Contributed equally

${ }^{* *}$ Contributed equally

Key words: meningioma, meningioma stroma, mesenchymal stem-like cells, microenvironment, perivascular area discovered. Immunohistochemical analyses indicated that $\mathrm{CD} 105^{+}$cells were closely associated with endothelial cells and pericytes in meningioma specimens. Our results established for the first time that cells similar to BM-MSCs exist in meningioma specimens. These cells, termed MS-MSLCs, could be one component of the meningioma cellular microenvironment.

\section{Introduction}

Brain cancer is one of the most devastating central nervous system pathologies and recent studies suggest that cancer stem cells (CSCs) are the most important oncogenic cells in brain cancer (1-3). Despite the importance of tumorigenic CSCs in the pathogenesis of brain cancer, increasing evidence supports a role for the microenvironment or stroma of brain cancer as an additional significant factor (4-7). Accordingly, the microenvironment including astrocytes, microglia and endothelial cells and the stroma, composed of non-neural cells, may support critical tumorigenic roles, such as initiation, progression $(1,2)$ and metastasis of cancers (5). The importance of the cancer microenvironment has received increased scrutiny since the 'seed and soil' hypothesis $(8)$ has been revisited $(9,10)$. Accordingly, we have taken a keen interest in the tumor microenvironment, especially mesenchymal stem-like cells (MSLCs), which resemble bone marrow mesenchymal stem cells (BM-MSCs), as components of the tumor microenvironment $(11,12)$.

Evidence suggests that glioblastomas are maintained by glioma CSCs (gCSCs) $(3,13,14)$ and, further, that understanding the microenvironment of gliomas is important for grasping glioma biology (15-18). After Lang et al first mentioned the isolation of mesenchymal stem cells (MSCs) from glioma specimens [Lang et al, Neuro-Oncol 9: abs. 596, 2007; Lang et al, J Clin Oncol 26 (Suppl 15): abs. 2001, 2008], MSLCs received considerable research attention and a recent series of studies have reported the isolation of MSCs/MSLCs from mouse normal brains (19), mouse orthotopic glioma specimens (11) and Korean glioma specimens (12). Furthermore, a very recent study investigated the relationship between gCSCs and glioma stroma MSLCs (GS-MSLCs) in glioblastoma (7). 
Similarly, we performed a series of studies examining the presence of gCSCs and their relationship $(3,6)$.

Although meningioma is one among the most common brain tumors also in Korea (20), little is known about meningioma cell biology. The recent successful isolation and characterization of CSCs from meningioma has provided a better understanding of meningioma biology (21-23). Components of the meningioma stroma are also likely important, as supported by previous studies (24-27).

Because meningioma is a mesenchymal tumor $(28,29)$, it is reasonable to suppose that meningiomas have a higher frequency of MSLCs. Despite the increased interest in meningioma to the best of our knowledge, there are no studies on meningioma stroma MSLCs (MS-MSLCs). In this study, we hypothesized that cells similar to BM-MSCs exist in meningioma specimen and tested this hypothesis based on cell morphology, differentiation potential, surface antigens and lack of oncogenicity. In addition, we sought to verify possible locations of MS-MSLCs.

\section{Materials and methods}

Single cell isolation and MS-MSLC culture. Specimens from patients with human meningioma were freshly obtained from the operating room with the approval of Institutional Review Boards of our institutes. Informed consent was provided according to the Declaration of Helsinki. Neuropathologists diagnosed these surgical specimens according to World health Organization (WHO) classification (30). Candidate MS-MSLCs were isolated from meningioma specimens within 60 min of meningioma removal using mechanical dissociation methods proven effective for MSC isolation from bone marrow (31,32), normal brain (19) and gliomas $(12,28)$. Briefly, surgical specimens were minced and dissociated with a scalpel in Dulbecco's modified Eagle's medium/ nutrient mixture F-12 (DMEM/F-12; Mediatech, Manassas, VA, USA) and then passed through a series of cell strainers with a $100-\mu \mathrm{m}$ nylon mesh (BD Falcon, Franklin Lakes, NJ, USA). Cell suspensions were washed twice in minimal essential medium- $\alpha$ (MEM $\alpha$; Mediatech, Herndon, VA, USA) and single-cell suspensions were placed in a $10-\mathrm{cm}^{2}$ cell culture dish at a density of $2 \times 10^{6} \mathrm{cell} / \mathrm{cm}^{2}$. These cells were cultured in complete MSC medium consisting of MEM $\alpha, 10 \%$ fetal bovine serum (FBS; Lonza, Basel, Switzerland), 2 mM L-glutamine (Mediatech) and antibiotic-antimycotic solution (100X, Gibco, Invitrogen Korea, Seoul, Korea). After 24 h, non-adherent cells were removed by washing twice with phosphate-buffered saline (PBS; Mediatech) and the adherent cells were cultured until they reached confluence. The cells were then trypsinized ( $0.25 \%$ trypsin with $0.1 \%$ EDTA) and sub-cultured at a density of 5,000 cells $/ \mathrm{cm}^{2}$. The cells were cultured continuously through 3-4 passages, consistent with their role as progenitor/ stem cells. Cell cultures were observed with an IX71 inverted phase-contrast microscope (Olympus, Tokyo, Japan) to determine their morphology. Images of cells were obtained at each passage using a DP70 Digital Microscope Camera (Olympus) equipped with DP Controller software (Olympus).

Flow cytometry analysis. To investigate the surface antigen expression profile, candidate MS-MSLCs were first counted and washed in PBS (Mediatech) by centrifugation, after which pellets were resuspended in fluorescent-activated cell sorting (FACS) buffer (PBS with 10\% FBS) at a concentration of $5 \times 10^{5}$ cells $/ 100 \mu \mathrm{l}$. These single-cell suspensions were incubated at $4^{\circ} \mathrm{C}$ for $30 \mathrm{~min}$ with phycoerythrin-, fluorescein isothiocyanate (FITC)-, Alexa Fluor 647-, or allophycocyanin-conjugated antibodies against CD105 $(0.25 \mu \mathrm{g} / 100 \mu \mathrm{l}$; eBioscience, San Diego, CA, USA), CD45 (5 $\mu \mathrm{g} / 100 \mu \mathrm{l}$; BD Pharmingen, San Diego, CA, USA), CD73 (5 $\mu \mathrm{g} / 100 \mu \mathrm{l}$; BD Pharmingen), CD90 (0.25 $\mu \mathrm{g} / 100 \mu \mathrm{l}$; eBioscience), CD31 $(0.5 \mu \mathrm{g} / 100 \mu \mathrm{l}$; eBioscience) and nerve/glial antigen 2 (NG2, $2.5 \mu \mathrm{g} / 100 \mu \mathrm{l}$; R\&D Systems, Minneapolis, MN, USA). All antibody solutions were prepared in FACS buffer. For the detection of NG2 proteoglycan, a FITC-conjugated secondary NG2 antibody (Millipore, Billerica, MA, USA) was used following primary antibody incubation (Santa Cruz Biotechnology, Inc., Santa Cruz, CA, USA). FACS analysis was performed using a FACS Vantage SE (BD Biosciences) flow cytometry system equipped with FlowJo software (Tree Star, Inc., Ashland, OR, USA) and 30,000 events were recorded for each sample. Because we merely sought to show the presence of MSLCs among heterogeneous cells instead of isolating a uniform population of MSCs (33), heterogeneous cell populations in which FACS showed that $>10 \%$ of cells expressed surface antigen were considered positive and those with $<5 \%$ by FACS were considered negative (12).

Mesenchymal differentiation. To determine the mesenchymal differentiation potential of candidate MS-MSLCs, we used a proven trilineage differentiation test identical to that described previously $(12,31,32)$. Briefly, we tested the capacity of candidate MS-MSLCs to differentiate along adipogenic, osteogenic and chondrogenic lineages. For adipogenic differentiation, MS-MSLCs were seeded in a 6-well plate at a density of $4 \times 10^{4}$ cells $/ \mathrm{cm}^{2}$ in complete MSC medium. At confluence, cell differentiation was induced with adipogenic differentiation medium from the adipogenic differentiation BulletKit (Lonza Walkersville, Walkersville, MD, USA). These cells were fed with fresh medium every 3-4 days for 3 weeks. In control experiments, cells were incubated for the same period of time in complete MSC medium. On day 21, the cells were washed in PBS (Mediatech) and fixed in 10\% formalin (Fisher Scientific, Fair Lawn, NJ, USA) for $1 \mathrm{~h}$ at room temperature. After fixation, the cells were rinsed with deionized water several times, after which of $60 \%$ isopropanol (Pharmco-AAPER, Brookfield, CT, USA) was added and cells were allowed to sit for $5 \mathrm{~min}$. Oil red $\mathrm{O}$ solution (Sigma) was then added to each well. After $5 \mathrm{~min}$, the cells were rinsed with deionized water and briefly counter-stained with hematoxylin (Sigma). For osteogenic differentiation, candidate MS-MSLCs were plated at a density of $3 \times 10^{4}$ cells $/ \mathrm{cm}^{2}$ in a 6 -well plate. The next day, the medium was replaced with osteogenic differentiation medium from the osteogenic differentiation BulletKit (Lonza Walkersville). These cells were fed with fresh medium every 3-4 days for 3 weeks. In control experiments, cells were incubated for the same period of time in complete MSC medium. On day 21, cell cultures were washed twice with PBS (Mediatech) and fixed in 70\% ice-cold ethanol (PharmcoAAPER) for $1 \mathrm{~h}$, followed by washing with deionized water. The cells were stained with $40 \mathrm{mM}$ Alizarin Red ( $\mathrm{pH} 4.2$; 


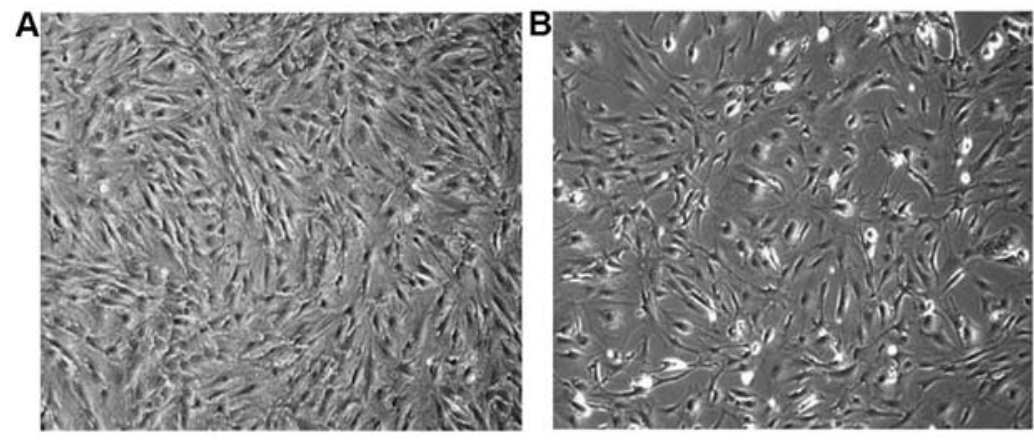

Figure 1. Morphology of MS-MSLCs from human meningiomas. Candidate MS-MSLCs from WHO grade II (A) and WHO grade I (B) meningiomas grew as spindle-shaped cells, like BM-MSCs.

Sigma) for $10 \mathrm{~min}$ at room temperature with rotation, followed by washing with deionized water five times. For chondrogenic differentiation, candidate MS-MSLCs were trypsinized and washed in serum-containing medium. Aliquots of $2.5 \times 10^{5}$ cells suspended in $0.5 \mathrm{ml}$ of medium were placed in $15-\mathrm{ml}$ conical polypropylene tubes (SPL, Pocheon, Gyeonggi, Korea). The cells were then gently centrifuged for $5 \mathrm{~min}$ at $150 \mathrm{x} \mathrm{g}$ and left at the bottom of the tubes, which were placed in an incubator with caps loosened to permit gas exchange. The cells formed small pellets that were cultured for 3 weeks in chondrogenic differentiation medium from the chondrogenic differentiation BulletKit (Lonza Walkersville) supplemented with $20 \mu \mathrm{g} / \mathrm{ml}$ of transforming growth factor (TGF)- $\beta 3$ (Ontogeny Research Products, Cambridge, MA, USA). Every 3-4 days, the cells were fed with fresh medium. In control experiments, the cells were incubated for the same period of time in complete MSC medium. These pellets were fixed in $10 \%$ formalin for $1 \mathrm{~h}$ at room temperature, then embedded in paraffin sections and stained with toluidine blue (Sigma) for proteoglycans and glycosaminoglycans.

Animal subjects. Four-to-eight-week-old male athymic nude mice (Central Laboratory Animal Inc., Seoul, Korea) were used to assess the tumorigenicity of candidate MS-MSLCs. Mice were housed in micro-isolator cages under sterile conditions and observed for $\geq 1$ week before study initiation to ensure proper health. Lighting, temperature and humidity were controlled centrally. All experimental procedures were approved by our Institutional Animal Care and Use Committee. The body weights of mice were checked daily. If body weight decreased by $>15 \%$ compared with the original body weight, mice were euthanized as proscribed by the approved protocol. The brain was dissected and placed in formalin for pathological studies.

Orthotopic meningiomaxenografting of candidate MS-MSLCs. Mice were anesthetized with a solution of Zoletil $(30 \mathrm{mg} / \mathrm{kg}$; Virbac Korea, Seoul, Korea) and xylazine (10 mg/kg; Bayer Korea, Seoul, Korea) delivered intraperitoneally. Candidate MS-MSLCs were implanted into the right frontal lobe of nude mice using a guide-screw system within the skull, as described previously (34). Mice received $5 \times 10^{5}$ candidate MS-MSLCs via a Hamilton syringe (Dongwoo Science Co., Seoul, Korea) inserted to a depth of $4.5 \mathrm{~mm}$. Each sample of candidate MSLCs was injected into three mice simultaneously using a multiple microinfusion syringe pump (Harvard Apparatus, Holliston, MA, USA) at a speed of $0.5 \mu \mathrm{l} / \mathrm{min}$, as previously described $(11,12,19,34,35)$. At least 180-200 days after injection, mouse brains were carefully removed, sectioned, stained with hematoxylin and eosin (H\&E) and examined for tumors.

Meningioma tissue preparation and immunofluorescence labeling. The possible location of MS-MSLCs in human meningioma specimens was investigated using double immunofluorescence labeling. Meningioma specimens were immediately removed and post-fixed in $4 \%$ paraformaldehyde at $4^{\circ} \mathrm{C}$ overnight. After dehydration with $30 \%$ sucrose in PBS, meningioma specimens were frozen with OCT compound (Sakura Finetek USA. Inc., Torrance, CA, USA) at $-80^{\circ} \mathrm{C}$. Frozen sections were processed for immunofluorescence labeling using goat anti-human CD105 (1:100; R\&D Systems), rabbit anti-human $\mathrm{CD} 31$ (1:50, an endothelial cell marker; Abcam, MA, USA) and rabbit anti-human NG2 antibodies (1:100, a pericyte marker; Millipore, Danvers, MA, USA). Alexa Fluor 488- and Alexa Fluor 555-conjugated goat antirabbit IgG antibodies (1:2,000; Invitrogen, CA, USA) were used as secondary antibodies. Samples were mounted in DAPI (4',6-diamidino-2-phenylindole)-containing Vectashield mounting medium (H-1200; Sunil Technopia, Seongnam, Korea) to stain nuclei and were examined under a fluorescence inverted microscope (IX71; Olympus) equipped with DP Controller software (Olympus).

Statistical analyses. Data are expressed as means \pm standard deviations. Survival curves for MS-MSLC-implanted mice were obtained using the Kaplan-Meier method. SPSS version 18.0KO software (SPSS Korea, Seoul, Korea) was used for calculations.

\section{Results}

Step 1: selection of MS-MSLCs by adherence to plastic. MS-MSLCs were obtained from a total of 20 meningioma specimens (10 WHO grade I and 10 WHO grade II) and grown under MSC culture conditions, as described previously $(12,31,32)$. Candidate MS-MSLCs with general properties of human BM-MSCs and MSLCs, characterized by their spindle shape and ability to adhere to plastic, were selected from WHO grade II (Fig. 1A) and grade I (Fig. 1B) meningiomas. Five of the ten WHO grade II meningioma samples and 2 of 
Table I. Step 1: selection of candidate MS-MSLCs based on adherence to plastic under MSC culture conditions.

\begin{tabular}{lccclcc}
\hline MS-MSLCs & Age & Sex & WHO grade & \multicolumn{1}{c}{ Pathology } & Adherence to plastic & Pass step 1 \\
\hline MS-MSLC0519 & 75 & M & II & Atypical meningioma & No & No \\
MS-MSLC0824 & 56 & F & II & Atypical meningioma & No & No \\
MS-MSLC0831 & 70 & F & II & Atypical meningioma & Yes & Yes \\
MS-MSLC0907 & 72 & F & II & Atypical meningioma & No & No \\
MS-MSLC1013 & 77 & M & II & Atypical meningioma & No & No \\
MS-MSLC1208 & 69 & M & II & Atypical meningioma & Yes & Yes \\
MS-MSLC0525 & 52 & F & II & Atypical meningioma & No & No \\
MS-MSLC0817 & 48 & F & II & Atypical meningioma & Yes & Yes \\
MS-MSLC0802 & 55 & M & II & Atypical meningioma & Yes & Yes \\
MS-MSLC1025 & 55 & F & II & Atypical meningioma & Yes & Yes \\
MS-MSLC0614 & 37 & M & I & Transitional meningioma & No & No \\
MS-MSLC0603 & 69 & F & I & Meningothelial meningioma & No & No \\
MS-MSLC0622 & 30 & F & I & Meningothelial meningioma & Yes & Yes \\
MS-MSLC0629 & 82 & F & I & Meningothelial meningioma & No & No \\
MS-MSLC0223 & 26 & M & I & Meningothelial meningioma & Yes & Yes \\
MS-MSLC0405 & 65 & M & I & Microcystic meningioma & No & No \\
MS-MSLC0928 & 51 & F & I & Secretory meningioma & No & No \\
MS-MSLC0608 & 46 & F & I & Meningothelial meningioma & No & No \\
MS-MSLC0627 & 20 & F & I & Meningothelial meningioma & No & No \\
MS-MSLC1112 & 37 & F & I & Fibrous meningioma & No & No \\
\hline
\end{tabular}

Table II. Step 2: selection of candidate MS-MSLCs based on surface marker expression. ${ }^{\text {a }}$

\begin{tabular}{lclccccccc}
\hline MS-MSLCs & $\begin{array}{c}\text { WHO } \\
\text { grade }\end{array}$ & \multicolumn{1}{c}{ Pathology } & $\begin{array}{c}\text { CD105 } \\
(\%)\end{array}$ & $\begin{array}{c}\text { CD90 } \\
(\%)\end{array}$ & $\begin{array}{c}\text { CD73 } \\
(\%)\end{array}$ & $\begin{array}{c}\text { CD45 } \\
(\%)\end{array}$ & $\begin{array}{c}\text { CD31 } \\
(\%)\end{array}$ & $\begin{array}{c}\text { NG2 } \\
(\%)\end{array}$ & $\begin{array}{c}\text { Pass } \\
\text { step 2 }\end{array}$ \\
\hline MS-MSLC0831 & II & Atypical meningioma & \multicolumn{7}{c}{ Failed to subculture } \\
MS-MSLC1208 & II & Atypical meningioma & 95.97 & 2.10 & 98.50 & 1.13 & 1.24 & 5.94 & No \\
MS-MSLC0802 & II & Atypical meningioma & 97.40 & 83.70 & 95.50 & 1.78 & 0.40 & 0.51 & Yes \\
MS-MSLC0817 & II & Atypical meningioma & 92.10 & 11.10 & 91.10 & 5.80 & 0.68 & 0.34 & Yes \\
MS-MSLC1025 & II & Atypical meningioma & 98.1 & 27.7 & 94.2 & 0.32 & 0.33 & 3.29 & Yes \\
MS-MSLC0223 & I & Meningothelial meningioma & 97.60 & 98.30 & 97.60 & 3.63 & 4.45 & 2.97 & Yes \\
MS-MSLC0622 & I & Meningothelial meningioma & 97.29 & 34.84 & 1.35 & 1.35 & 1.19 & 1.61 & No \\
\hline
\end{tabular}

${ }^{a}$ Cells that were positive for CD105, CD90 and CD73 and negative for CD45, CD31 and NG2 were chosen for further tests of differentiation ability.

the 10 WHO grade I meningioma samples passed step 1 and were selected for characterization (Table I). Although the proportion of spindle-shaped, adherent cells in each of these selected specimens was different, their morphology showed little difference between WHO grade I and II.

Step 2: selection of MS-MSLCs based on surface antigen expression. Flow cytometry analysis was used to assess surface antigen expression in spindle-shaped cells that adhered to plastic under MSC/MSLC culture conditions. Although there are no specific pathognomonic markers for human BM-MSCs, it is generally agreed that CD105, CD90 and CD73 are posi- tive markers and CD45 is negative marker for most MSLCs $(12,32,33)$. Using these criteria, we tested whether candidate cells are MS-MSLCs (Fig. 2). Because MSLCs from mice normal brains (19), glioma xenografts (11) and Korean glioma specimens (12) are found around vessels, CD31, a marker of endothelial cells and NG2, a marker of pericyte were additionally used to discriminate MS-MSLCs and vessel-related cells. MS-MSLCs were negative for CD31 and NG2 (Fig. 2), as expected for these non-endothelial, non-pericyte cells. Of the meningioma specimens that passed step 1, three of five WHO grade II and one of two WHO grade I specimens showed proper surface antigen expression (Table II). 

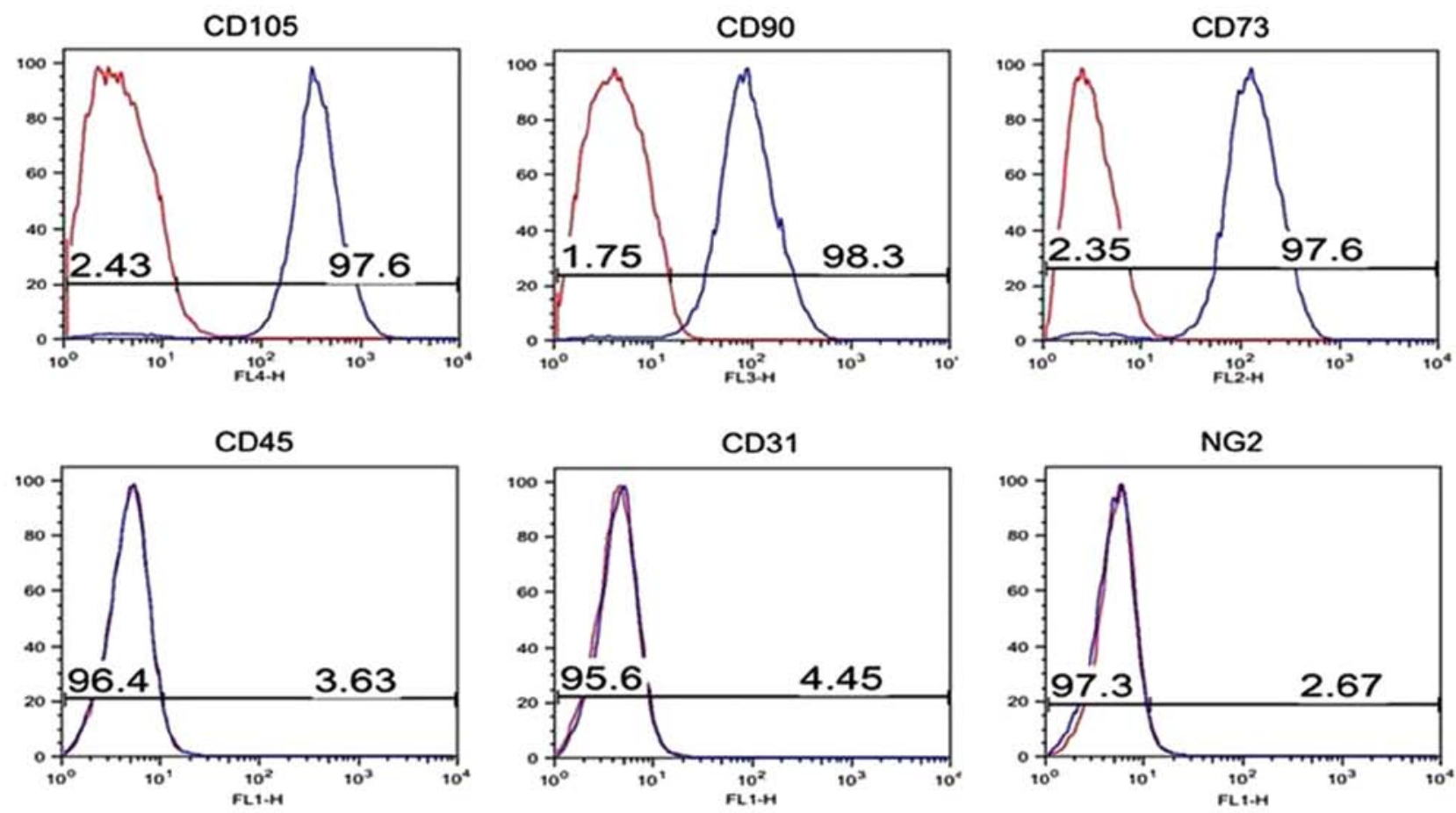

Figure 2. Selection of candidate MS-MSLCs based on surface antigen expression by flow cytometric analysis. The mesenchymal markers CD105, CD90 and CD73 were chosen as positive markers and CD45 (leukocytes), CD31 (endothelial cells) and NG2 (pericytes) were used as negative markers.
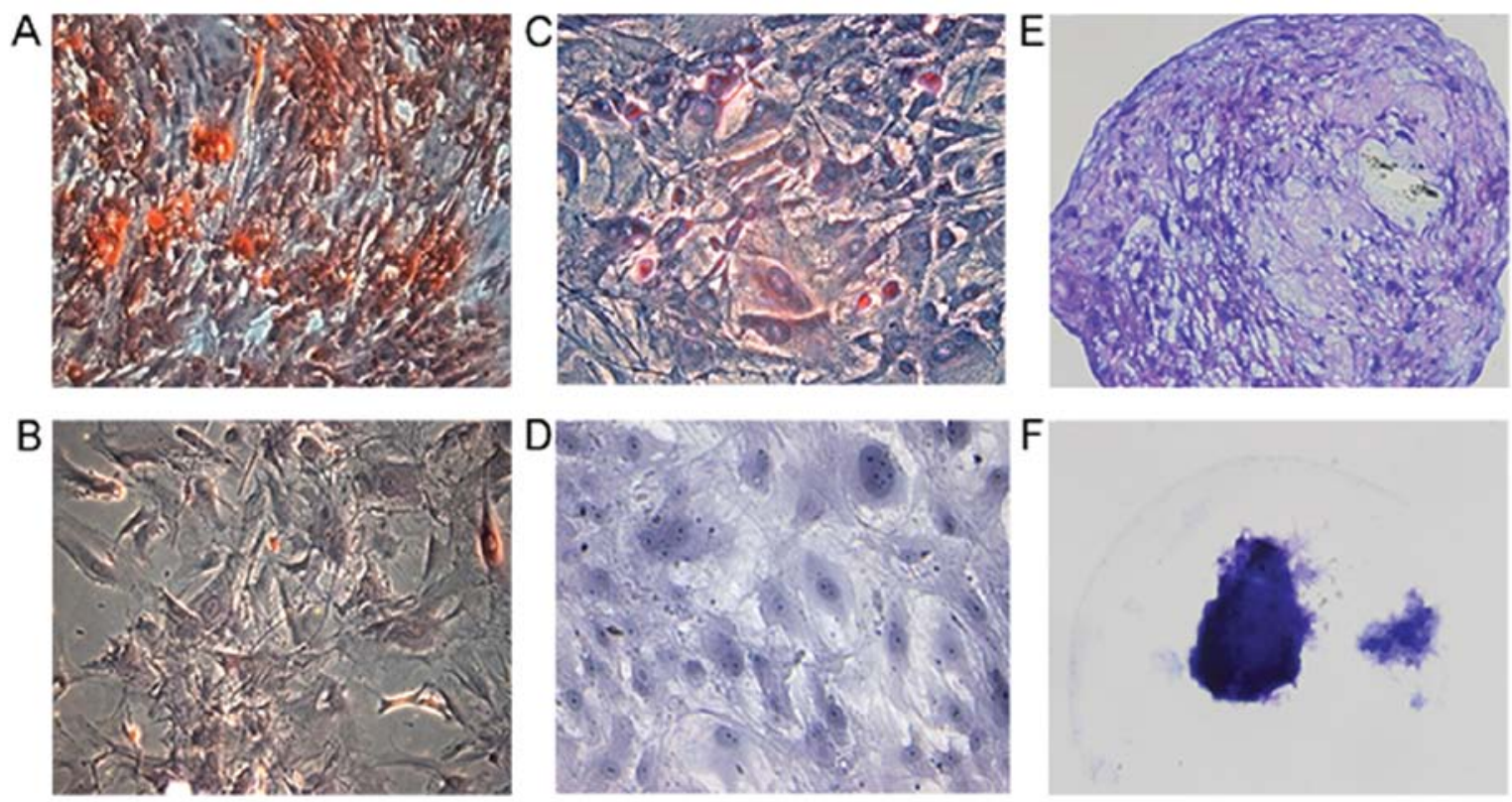

Figure 3. Trilineage mesenchymal differentiation potential of MS-MSLCs. (A) Differentiation of candidate MS-MSLCs into osteocytes, as evidenced by calcium deposition (Alizarin Red staining), was observed under osteogenic conditions. (B) Alizarin Red staining revealed that non-induced candidate MS-MSLCs (control) were negative for osteogenesis. (C) Differentiation of candidate MS-MSLCs into adipocytes, as evidenced by the presence of intracellular lipid droplets (Oil Red O staining), was observed under adipogenic differentiation conditions. (D) Oil Red O staining revealed that non-induced candidate MS-MSLCs were negative for adipogenesis. (E) Differentiation of candidate MS-MSLCs into chondrocytes, as evidenced by the presence of proteoglycans and glycosaminoglycans (toluidine blue staining), was observed under chondrogenic differentiation conditions. (F) Toluidine blue staining revealed that non-induced MS-MSLCs were negative for chondrogenesis.

Step 3: selection of MS-MSLCs based on mesenchymal differentiation. MSCs/MSLCs exhibit trilineage - osteocyte, adipocyte and chondrocyte - differentiation capacity $(11,12,19,33)$. To validate the mesenchymal trilineage differentiation potential of MS-MSLCs, we tested candidate cells that passed steps 1 and 2 for their ability to differentiate into osteocytes, adipocytes and chondrocytes when cultured in induction medium (Fig. 3A, C and E). Differentiation into only two of the three cell types meant failure to pass step 3 . No trilineage differentiation was observed in control medium 


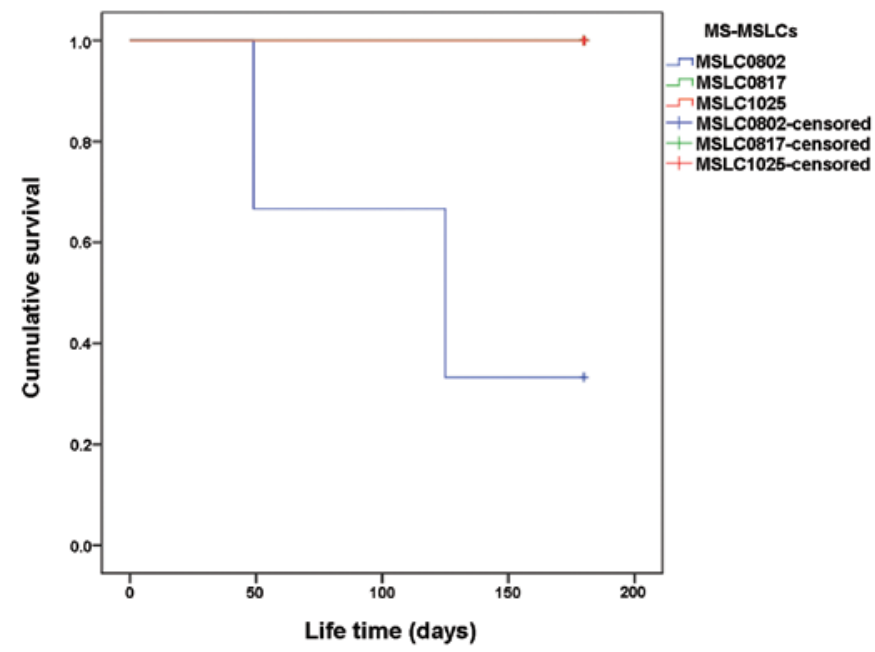

Figure 4. Non-tumorigenicity of intracranially implanted MS-MSLCs in nude mice. Survival curves demonstrate that engrafted nude mice implanted with MS-MSLC0817 or MS-MSLC1025 cells survived for $>6$ months. Although nude mice implanted with the MS-MSLC0802 cell group died earlier, death was not caused by tumors but was rather attributable to infection.

(Fig. 3B, D and F). Among the selected candidate MS-MSLCs, only WHO grade II meningioma cells satisfied the criterion of trilineage differentiation potential (Table III).

Step 4: selection of MS-MSLCs based on in vivo non-tumorigenicity. Unlike CSCs, MSCs/MSLCs are not tumorigenic in vivo. To satisfy this criterion, most mice intracranially implanted with candidate MS-MSLCs that passed steps 1, 2 and 3 should survive for more than 6 months. Tests of the three candidate MS-MSLCs from WHO grade II meningioma samples that passed steps 1,2 and 3 showed that mice implanted with candidate MS-MSLCs from two samples
(MS-MSLC0817 and MS-MSLC1025) survived for $>6$ months (Fig. 4), whereas those implanted with the third sample (MS-MSLC0802) died 4 months later (Table IV). Notably, however, mice in the group implanted with MS-MSLC0802 meningioma cells that failed to survive $>4$ months died from infection and not because of a tumor. Accordingly, two groups of MS-MSLCs (MS-MSLC0817 and MS-MSLC1025) isolated from meningioma specimens passed step 4, the final test for MS-MSLC selection, convincingly demonstrating no tumorigenicity or general toxicity (Table V).

Immunofluorescence detection of CD31, NG2 and CD105. The results of steps 1-4 corroborate the hypothesis that MSLCs exist in meningioma specimens, although the question of where MS-MSLCs are located remained. Previous studies of MSLCs in normal mouse brains (19), mouse glioma xenografts (11) and Korean glioma specimens (12) have suggested that these cells were located in a perivascular site. To verify that MS-MSLCs might also be located in a perivascular niche, we analyzed meningioma specimens for expression of the markers CD105, CD31 and NG2 by immunofluorescence. CD105, a surface marker present in most MSCs/MSLCs, was selected for establishing the presence of MS-MSLCs. To determine whether CD105-positive cells were near endothelial cells, we performed double-immunofluorescence labeling for the endothelial cell markers, CD31. Histological analyses suggested that some CD105-positive cells were closely associated with CD31-positive cells (Fig. 5A). To determine whether CD105-positive cells were associated with pericytes, we performed double-immunofluorescence labeling for CD105 and the pericyte marker NG2. Similar to the results obtained with CD105 and CD31 double-immunofluorescence labeling, some CD105-positive cells were intimately associated with NG2-positive cells (Fig. 5B). Accordingly, we infer that some CD105-positive candidate MS-MSLCs are located in the
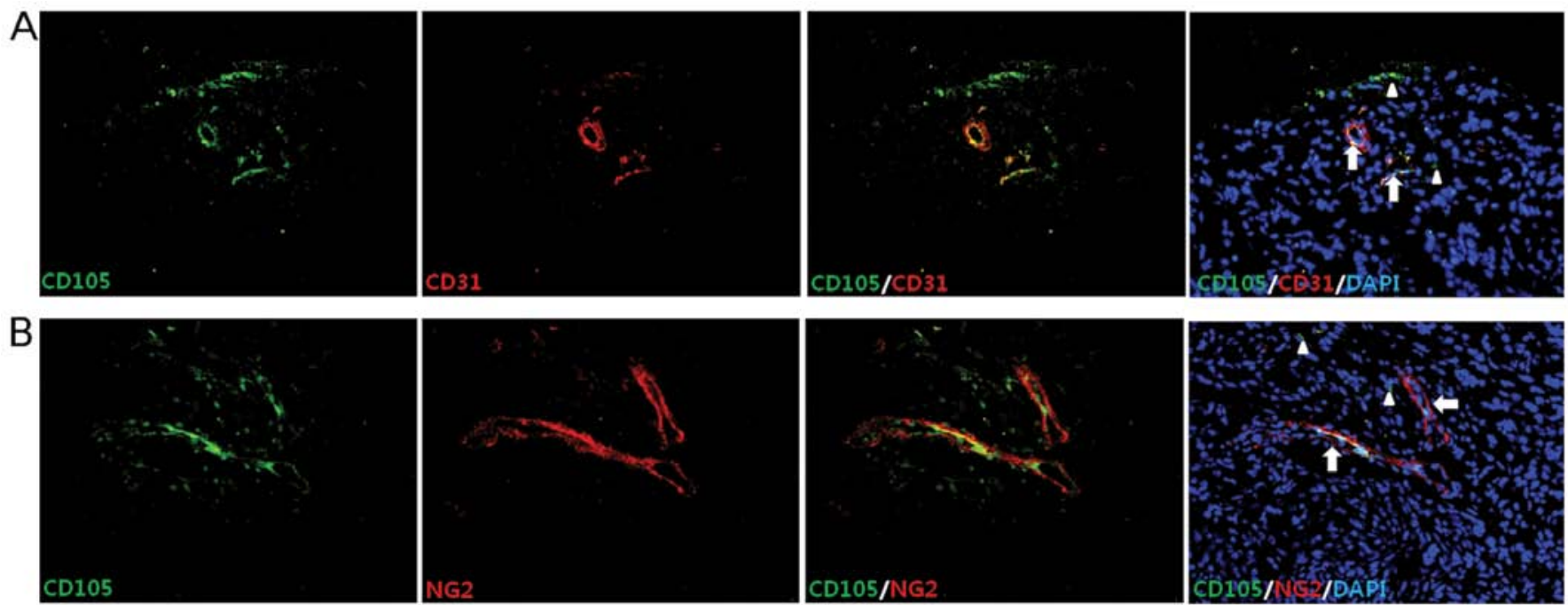

Figure 5. Double-immunofluorescence labeling (CD105/CD31 and CD105/NG2) of meningioma specimens. Green fluorescence labeling indicates CD105positive cells, some of which may be MS-MSLCs. Red fluorescence labeling indicates CD31-positive (endothelial cells) or NG2-positive (pericytes) cells Nuclei are counterstained with DAPI (blue). (A) CD105/CD31 double staining demonstrates the close relationship between CD105-positive and CD31-positive cells (arrows), although not all CD105-positive cells are associated with CD31-positive cells (arrowheads). (B) CD105/NG2 double staining of demonstrates the close relationship between CD105-positive and NG2-positive cells (arrows), although but not all CD105-positive cells are associated with NG2-positive cells (arrowheads). 
Table III. Step 3: selection of MS-MSLCs based on in vitro mesenchymal differentiation. ${ }^{\mathrm{a}}$

\begin{tabular}{lcccccc}
\hline MS-MSLCs & WHO grade & Pathology & Osteogenesis & Adipogenesis & Chondrogenesis & Pass step 3 \\
\hline MS-MSLC0802 & II & Atypical meningioma & Yes & Yes & Yes & Yes \\
MS-MSLC0817 & II & Atypical meningioma & Yes & Yes & Yes & Yes \\
MS-MSLC1025 & II & Atypical meningioma & Yes & Yes & Yes & Yes \\
MS-MSLC0223 & I & Meningothelial meningioma & Yes & No & Yes & No \\
\hline
\end{tabular}

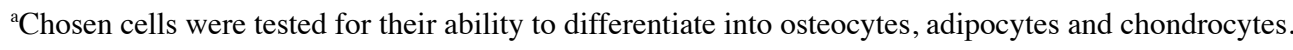

Table IV. Step 4: selection of candidate MS-MSLCs based on tumorigenicity.

\begin{tabular}{lcccc}
\hline MS-MSLCs & WHO grade & Pathology & Tumorigenesis & Pass step 4 \\
\hline MS-MSLC0802 $^{\mathrm{a}}$ & II & Atypical meningioma & No & No \\
MS-MSLC0817 & II & Atypical meningioma & No & Yes \\
MS-MSLC1025 & II & Atypical meningioma & No & Yes \\
\hline
\end{tabular}

${ }^{a}$ The nude mice implanted with this cell population died not because of tumor but infection.

Table V. Final success rate for isolation of MS-MSLCs according to selection step (1-4).

\begin{tabular}{|c|c|c|c|c|c|}
\hline Pathology & $\begin{array}{c}\text { Step 1 } \\
\text { Plastic } \\
\text { adherence \% }\end{array}$ & $\begin{array}{c}\text { Step } 2 \\
\text { Surface antigen } \\
\text { expression } \%\end{array}$ & $\begin{array}{c}\text { Step } 3 \\
\text { Mesenchymal } \\
\text { differentiation \% }\end{array}$ & $\begin{array}{c}\text { Step } 4 \\
\text { No tumori- } \\
\text { genesis \% }\end{array}$ & $\begin{array}{c}\text { Final success rate } \\
\text { of MS-MSLCs } \\
\text { isolation } \%\end{array}$ \\
\hline WHO grade II & $50(5 / 10)$ & $60(3 / 5)$ & $100(3 / 3)$ & $66.7(2 / 3)$ & $20(2 / 10)$ \\
\hline Atypical MNG & $50(5 / 10)$ & $60(3 / 5)$ & $100(3 / 3)$ & $66.7(2 / 3)$ & $20(2 / 10)$ \\
\hline WHO grade I & $20(2 / 10)$ & $50(1 / 2)$ & $0(0 / 1)$ & $0 \quad(0 / 1)$ & $0(0 / 10)$ \\
\hline Meningothelial MNG & $60(3 / 5)$ & $50(1 / 2)$ & $0(0 / 1)$ & $0 \quad(0 / 1)$ & $0(0 / 2)$ \\
\hline Microcystic MNG & $0(0 / 1)$ & & & & $0(0 / 1)$ \\
\hline Secretory MNG & $0(0 / 1)$ & & & & $0(0 / 1)$ \\
\hline
\end{tabular}

MNG, meningioma.

perivascular niche (Fig. 5, arrows). However, not all CD105positive cells were found near vessels. These cells may be niche-independent cells (Fig. 5, arrowheads).

\section{Discussion}

Considerable recent evidence supports the presence of MSCs in various human tissues $(19,36)$. Other studies have also reported the existence of MSCs or MSLCs in the stroma of brain and other tumors $(6,19,37-39)$, although little information about the function of these cells is available. In the present study, we successfully isolated MS-MSLCs from meningioma specimens with plastic adherence properties (Fig. 1 and Table I) and a surface antigen profile (Fig. 2 and Table II) similar to those of BM-MSCs. In addition, these cells exhibited mesenchymal trilineage differentiation capacity (Fig. 3 and Table III) and the absence of tumorigenicity (Fig. 4 and Table IV). We also found evidence for localization of a subset of these MS-MSLCs to perivascular areas.

Ultimately, MS-MSLCs that satisfied all four criteria (adherence to plastic, surface antigen expression, mesenchymal differentiation and non-tumorigenicity) were isolated from 2 of 10 WHO grade II meningioma specimens (20\%). In a previous study, Korean GS-MSLCs (KGS-MSLCs) were isolated from 1 of 5 WHO grade II Korean glioma specimens (20\%), but not from WHO grade I specimens (0/1) (12). Consistent with this, both meningioma specimens that yielded MS-MSLCs in the present study were WHO grade II (Table V). Because meningiomas are mesenchymal tumors $(28,29)$ and gliomas are neuroepithelial in origin, we initially anticipated that the rate of isolation of MSLCs from meningiomas would be higher; however, this turned out not to be the case. Despite the fact that meningiomas and gliomas are histologically different tumors, MSLC isolation rates were similar (20\%) and only WHO 
grade II tumors yielded MSLCs that satisfied all criteria. Thus, although sample sizes were small (e.g., only 1 WHO grade I and 5 grade II gliomas in the previous study), the results of our study taken together with the previous report (12) suggest that the frequency of MSLC isolation depends on WHO grade rather than cancer type.

Malignant meningiomas are highly aggressive and easily recur after surgical treatment (40), so understanding the mechanism of meningioma recurrence is highly important. Studies have shown that arachnoid cells, which share similar properties with meningioma cells, are a significant factor in recurrence (41) demonstrating, for example, that arachnoid membranes containing arachnoid cells and clusters of cancer cells are closely related to meningioma recurrence (42). These studies suggest that cancer cells near arachnoid cells and a perivascular site might follow the mechanism by which arachnoid cells preferentially locate around perivascular areas and penetrate into the brain $(41,43)$. The results of our double-immunofluorescence labeling for CD31, NG2 and CD105 might be consistent with localization of MS-MSLCs in a vascular niche, possibly indicating that MSLCs follow a mechanism similar to that of penetrating arachnoid cells, although our data do not provide direct evidence for this, such a mechanism could be a crucial determinant of meningioma recurrence. We are currently following the progression-free survival of the two different patient groups: those from whom MS-MSLCs could be isolated and those from whom they could not. These follow-up observations could show the prognostic value of MS-MSLCs.

Although the origin of meningioma is unclear, it is believed that arachnoid cells are the most likely source (44); thus, most meningiomas occur near cerebral meninges. To evaluate in vivo tumorigenicity, we used an intracranial meningioma mouse model, implanting candidate MS-MSLCs into the right frontal lobe of a nude mouse. Although the use of this xenograft model might be questioned because cells were intracranially injected, these cells were usually injected into the subdural space near cerebral meninges, where meningiomas are typically found. Because, in the intracranial xenograft model system, tumors form within the brain, they might show some differences in characteristics. However, others have tested the tumorigenesis of WHO grade II meningioma using intracranial injection mouse models (21). Accordingly, we adopted this intracranial xenograft system (21) to evaluate the in vivo tumorigenicity of meningioma-derived MSLCs (Fig. 4).

CD105 (endoglin) is an endothelial cell protein that binds TGF- $\beta$ (45). CD105 is also expressed on immature blood vessels, whereas CD31 is an endothelial cell marker that is not expressed on immature vessels. For this reason, CD105 can be used as a single marker to verify angiogenesis $(46,47)$. However, endothelial cells are not the only cells that express CD105; BM-MSCs are also highly CD105-positive. Hence, CD105 is frequently used as a marker for MSCs/MSLCs and a tool for isolating MSLC populations from specimens $(12,48,49)$. Although CD105 is used to screen for MSCs, there is no single marker for these cells. Because of this, it is impossible to confirm that CD105-positive cells are MSCs. Following the minimal requirement for defining MSCs (33), we used the surface markers CD90, CD73 and CD45 (12) to define MSLCs (Fig. 2). To distinguish MS-MSLCs from endothelial cells and pericytes, we used CD31, a marker of endothelial cells and NG2, a marker of pericytes, as negative surface markers (Table II).

The results of double-immunofluorescence labeling for CD31, NG2 and CD105 in this study showed that CD105positive cells were located in two different sites (Fig. 5). Some clusters of these cells were situated near endothelial cells (Fig. 5A, arrows) and pericytes (Fig. 5B, arrows), whereas others were located inside the meningioma stroma (Fig. 5, arrowheads). This outcome indirectly suggests the possible location of MS-MSLCs as the meningioma stroma and perivascular areas. Although our study is the first to show the successful isolation and characterization of MSLCs from meningioma specimens, there is no direct method to definitively establish their location. Another question is the uncertain origin of putative MS-MSLCs near blood vessels. These cells could be innate meningioma stroma MSLCs or circulating MSLCs derived from bone marrow. Resolving this question will require further studies to validate the origin of cells situated near the vascular niche.

Recent studies demonstrated the isolation and characterization of meningioma stem-like cells $(21,23)$. The relationship between these so-called meningioma CSCs (mCSCs) and the MS-MSLCs isolated from meningiomas and described in the present study is not clear. One report on gliomas suggests a relationship between gCSCs and GS-MSLCs (7). In that study, GS-MSLCs were proposed to influence gCSCs and make gliomas more aggressive by promoting angiogenesis (7). On the basis of this relationship, we postulate that MS-MSLCs are related to mCSCs, although further study will be required to verify this hypothesis. Mesenchymal tumors share a molecular signature with MSCs (50), indicating a close relationship between meningiomas and mesenchymal molecular signatures. This suggests that the mesenchymal molecular features of meningiomas might be derived from MSLCs in the meningioma stroma. The intriguing possibility of a connection between the mesenchymal molecular signatures of meningiomas and MS-MSLCs, which was not directly addressed in the present study, is currently under investigation in our laboratory.

According to the 'seed and soil' hypothesis (8), CSCs are considered the seed and the tumor microenvironment is considered the soil $(9,10)$. Within the tumor, CSCs are identified by virtue of their self-renewal, differentiation and tumorigenicity in orthotopic xenografts $(3,13,14)$, whereas other cells in the tumor microenvironment might be thought of as elements that are significant for the biologic behavior of CSCs $(4,7)$. The seed and soil hypothesis is crucially important for understanding the mechanism of metastasis $(8,9)$. According to our studies, MS-MSLCs might be considered an important part of meningiomas and could be a new cell source of the meningioma microenvironment. In addition, these MS-MSLCs might be the key to unlocking the relationship between mCSCs and meningioma stroma cells. Investigating the biological relationship between MS-MSLCs and mCSCs in the context of the seed and soil concept is a fertile avenue for future research.

\section{Acknowledgements}

This research was supported by the Basic Science Research Program through the National Research Foundation of Korea 
(NRF) funded by the Ministry of Education, Science and Technology (NRF-2013R1A1A2006427) and a grant from the National R\&D Program for Cancer Control, Ministry for Health, Welfare and Family Affairs, Republic of Korea (1020340).

\section{References}

1. Fomchenko EI and Holland EC: Stem cells and brain cancer. Exp Cell Res 306: 323-329, 2005.

2. Galderisi U, Cipollaro M and Giordano A: Stem cells and brain cancer. Cell Death Differ 13: 5-11, 2006.

3. Kong BH, Park NR, Shim JK, et al: Isolation of glioma cancer stem cells in relation to histological grades in glioma specimens. Childs Nerv Syst 29: 217-229, 2013

4. Liotta LA and Kohn EC: The microenvironment of the tumourhost interface. Nature 411: 375-379, 2001.

5. Xouri $\mathrm{G}$ and Christian S: Origin and function of tumor stroma fibroblasts. Semin Cell Dev Biol 21: 40-46, 2010.

6. Shin GY, Shim JK, Lee JH, et al: Changes in the biological characteristics of glioma cancer stem cells after serial in vivo subtransplantation. Childs Nerv Syst 29: 55-64, 2013.

7. Kong BH, Shin HD, Kim SH, et al: Increased in vivo angiogenic effect of glioma stromal mesenchymal stem-like cells on glioma cancer stem cells from patients with glioblastoma. Int J Oncol 42: 1754-1762, 2013.

8. Paget S: The distribution of secondary growths in cancer of the breast. Lancet 133: 571-573, 1889.

9. Fidler IJ and Poste G: The 'seed and soil' hypothesis revisited. Lancet Oncol 9: 808, 2008

10. Mendoza M and Khanna C: Revisiting the seed and soil in cancer metastasis. Int J Biochem Cell Biol 41: 1452-1462, 2009.

11. Kim SM, Kang SG, Park NR, et al: Presence of glioma stroma mesenchymal stem cells in a murine orthotopic glioma model Childs Nerv Syst 27: 911-922, 2011.

12. Kim YG, Jeon S, Sin GY, et al: Existence of glioma stroma mesenchymal stemlike cells in Korean glioma specimens. Childs Nerv Syst 29: 549-563, 2013.

13. Singh SK, Clarke ID, Terasaki M, et al: Identification of a cancer stem cell in human brain tumors. Cancer Res 63: 5821-5828, 2003.

14. Singh SK, Hawkins C, Clarke ID, et al: Identification of human brain tumour initiating cells. Nature 432: 396-401, 2004.

15. Hoelzinger DB, Demuth T and Berens ME: Autocrine factors that sustain glioma invasion and paracrine biology in the brain microenvironment. J Natl Cancer Inst 99: 1583-1593, 2007.

16. Oliver L, Olivier C, Marhuenda FB, Campone M and Vallette FM: Hypoxia and the malignant glioma microenvironment: regulation and implications for therapy. Curr Mol Pharmacol 2: 263-284, 2009.

17. Kanamori M, Kawaguchi T, Berger MS and Pieper RO Intracranial microenvironment reveals independent opposing functions of host alphaVbeta3 expression on glioma growth and angiogenesis. J Biol Chem 281: 37256-37264, 2006

18. Stewart PA, Farrell CL and Del Maestro RF: The effect of cellular microenvironment on vessels in the brain. Part 1: vessel structure in tumour, peritumour and brain from humans with malignant glioma. Int J Radiat Biol 60: 125-130, 1991.

19. Kang SG, Shinojima N, Hossain A, et al: Isolation and perivascular localization of mesenchymal stem cells from mouse brain. Neurosurgery 67: 711-720, 2010.

20. Lee CH, Jung KW, Yoo H, Park S and Lee SH: Epidemiology of primary brain and central nervous system tumors in Korea J Korean Neurosurg Soc 48: 145-152, 2010.

21. Hueng DY, Sytwu HK, Huang SM, Chang C and Ma HI: Isolation and characterization of tumor stem-like cells from human meningiomas. J Neurooncol 104: 45-53, 2011.

22. Rath P, Miller DC, Litofsky NS, et al: Isolation and characterization of a population of stem-like progenitor cells from an atypical meningioma. Exp Mol Pathol 90: 179-188, 2011.

23. Hu D, Wang X, Mao Y and Zhou L: Identification of CD105 (endoglin)-positive stem-like cells in rhabdoid meningioma. J Neurooncol 106: 505-517, 2012.

24. Mosnier JF, Perret AG, Scoazec JY and Brunon J: Expression of beta 2 integrins and macrophage-associated antigens in meningeal tumours. Virchows Arch 436: 131-137, 2000.

25. Kimura Y, Matsumae M and Tsutsumi Y: Pericellular deposition of basement membrane material in myxoid meningioma: immunohistochemical evidence for unbalanced production of type IV collagen and laminin. Pathol Int 48: 53-57, 1998.
26. Shamah SM, Alberta JA, Giannobile WV, et al: Detection of activated platelet-derived growth factor receptors in human meningioma. Cancer Res 57: 4141-4147, 1997.

27. Nystrom SH: Fine structure of tumour stroma and blood vessel stroma in human supratentorial menigioma. Nature 194: 587-588, 1962.

28. Majumdar K, Mandal S, Thakkar R, Saran RK and Srivastava AK: Meningeal osteochondroma simulating meningioma with metaplastic change: a rare golf-ball-like lesion of non-meningothelial mesenchymal origin. Brain Tumor Pathol: Mar 2, 2013 (Epub ahead of print)

29. Celebre A, Wu MY, Danielson B, et al: Anaplastic meningioma with extensive single-cell infiltration: a potential role for epithelial-mesenchymal transformation in the progression of a meningothelial tumour? Histopathology 62: 1111-1114, 2013.

30. Louis DN, Ohgaki H, Wiestler OD, et al: The 2007 WHO classification of tumours of the central nervous system. Acta Neuropathol 114: 97-109, 2007.

31. Mareschi K, Biasin E, Piacibello W, Aglietta M, Madon E and Fagioli F: Isolation of human mesenchymal stem cells: bone marrow versus umbilical cord blood. Haematologica 86: 1099-1100, 2001.

32. Lennon DP and Caplan AI: Isolation of human marrow-derived mesenchymal stem cells. Exp Hematol 34: 1604-1605, 2006.

33. Dominici M, Le Blanc K, Mueller I, et al: Minimal criteria for defining multipotent mesenchymal stromal cells. The International Society for Cellular Therapy position statement. Cytotherapy 8: 315-317, 2006.

34. Lal S, Lacroix M, Tofilon P, Fuller GN, Sawaya R and Lang FF: An implantable guide-screw system for brain tumor studies in small animals. J Neurosurg 92: 326-333, 2000.

35. Nakamizo A, Marini F, Amano T, et al: Human bone marrowderived mesenchymal stem cells in the treatment of gliomas. Cancer Res 65: 3307-3318, 2005.

36. da Silva Meirelles L, Chagastelles PC and Nardi NB: Mesenchymal stem cells reside in virtually all post-natal organs and tissues. J Cell Sci 119: 2204-2213, 2006.

37. El-Haibi CP and Karnoub AE: Mesenchymal stem cells in the pathogenesis and therapy of breast cancer. J Mammary Gland Biol Neoplasia 15: 399-409, 2010.

38. Karnoub AE, Dash AB, Vo AP, et al: Mesenchymal stem cells within tumour stroma promote breast cancer metastasis. Nature 449: 557-563, 2007.

39. Hall B, Andreeff M and Marini F: The participation of mesenchymal stem cells in tumor stroma formation and their application as targeted-gene delivery vehicles. Handb Exp Pharmacol 180: 263-283, 2007.

40. Feigin I: Mixed mesenchymal tumors: meningioma and nerve sheath tumor. J Neuropathol Exp Neurol 37: 459-470, 1978.

41. Ng HK, Tse CC and Lo ST: Meningiomas and arachnoid cells: an immunohistochemical study of epithelial markers. Pathology 19: 253-257, 1987.

42. Kamitani H, Masuzawa H, Kanazawa I and Kubo T: Recurrence of convexity meningiomas: tumor cells in the arachnoid membrane. Surg Neurol 56: 228-235, 2001.

43. Lopes CA and Mair WG: Tubular structures in arachnoid cells. Acta Neuropathol 27: 363-368, 1974.

44. Ohnishi Y, Iwatsuki K, Morii E, et al: Histopathological study of spinal meningioma originating from the arachnoid villi. Brain Tumor Pathol 28: 77-81, 2011.

45. Cheifetz S, Bellon T, Cales C, et al: Endoglin is a component of the transforming growth factor-beta receptor system in human endothelial cells. J Biol Chem 267: 19027-19030, 1992.

46. Behrem S, Zarkovic K, Eskinja N and Jonjic N: Endoglin is a better marker than CD31 in evaluation of angiogenesis in glioblastoma. Croat Med J 46: 417-422, 2005.

47. Barresi V and Barresi G: Endoglin: a marker of neoplasias or rather of neo-angiogenesis? Head Neck 32: 970-971, 2010.

48. Yen BL, Huang HI, Chien CC, et al: Isolation of multipotent cells from human term placenta. Stem Cells 23: 3-9, 2005.

49. Crisan M, Chen CW, Corselli M, Andriolo G, Lazzari L and Peault B: Perivascular multipotent progenitor cells in human organs. Ann NY Acad Sci 1176: 118-123, 2009.

50. Galie M, Konstantinidou G, Peroni D, et al: Mesenchymal stem cells share molecular signature with mesenchymal tumor cells and favor early tumor growth in syngeneic mice. Oncogene 27: 2542-2551, 2008. 